\title{
Design of HIS-backed Miniaturized Cross Slotted Antenna for Circular Polarization Using Modal Analysis: A Novel Approach
}

\author{
Arka BHATTACHARYYA ${ }^{1}$, Kaushik PATRA ${ }^{2}$, Bhaskar GUPTA $^{1}$ \\ ${ }^{1}$ Dept. of ETCE, Jadavpur University, 188, Raja SC Mullick Road, Kolkata-700032, India \\ ${ }^{2}$ Dept. of ECE, Institute of Engineering \& Management, Street Number 18, EP Block, Sector V, Kolkata-700091, India
}

arkabhattacharyya47@gmail.com, kaushikpatra294@gmail.com, gupta_bh@yahoo.com

Submitted March 20, 2020 / Accepted November 26, 2020

\begin{abstract}
This work presents a novel design technique of a circularly polarized miniaturized cross slotted antenna backed by a High Impedance Surface using substructure based Characteristic Mode analysis. The electromagnetic behavior of the antenna and the HIS is studied while the two structures are in close vicinity, with no air gap isolation between them. Such problem of proximity induced near field interaction of the finite HIS with the antenna has rarely been systematically attempted in literature. The chosen operating frequency of the cross slotted antenna is synchronized with the frequency of high impedance operation of the HIS by analysing the structure. This work explains how the characteristics of HIS can be determined when placed at the near electromagnetic field of an antenna. Circularly polarized radiation is obtained at $2.45 \mathrm{GHz}$ with a 3-dB axial ratio bandwidth close to $1.46 \%$ with a 10-dB impedance bandwidth of $4.4 \%$. The overall size of the designed antenna is $0.23 \lambda_{0} \times 0.23 \lambda_{0} \times 0.039 \lambda_{0}$ including the substrate dimensions at $2.45 \mathrm{GHz}$ ( $\lambda_{0}$ is the free space wavelength). The area reduction of the complete antenna and only the slotted patch is achieved as $78 \%$ and $88 \%$ respectively, as compared to the fundamental mode half wavelength antenna at this frequency.
\end{abstract}

\section{Keywords}

High impedance surface (HIS), characteristic modes $(\mathrm{CM})$, circular polarization $(\mathrm{CP})$, miniaturization, cross slotted antenna

\section{Introduction}

Bidirectional radiation pattern of slotted ground plane antennas is unwanted in some practical applications. Especially in case of RFID tags, wearable and implantable antennas - a strictly unidirectional pattern is required to ensure reliable data transmission, good antenna efficiency and also to avoid health hazards due to backward radiation absorption into human body. In these applications, High Impedance Surface (HIS) backed slotted antennas are preferred due to the in-phase reflection produced by the HIS. This results in constructive interference between the HIS-reflected field and the radiated field of the slotted antenna in far field. However, the design of HIS requires a bigger space because of the periodicity of the building blocks of the meta-surface. Present day communication systems require smaller antennas to be integrated inside chips or to be effectively embedded into wearable devices. These requirements pose more constraints to the overall design of the antenna. The forced truncation of HIS causes the antenna to have a significant back radiation due to edge diffraction. Also, the theoretical operating frequencies and reflection phases of radiated fields then are affected due to the truncation of the HIS.

The other very important challenge of designing HIS backed slotted antennas is to make the overall structure low profile or electrically thin. As soon as the antenna is brought closer to the HIS, the near field of the antenna starts interacting drastically with the HIS. The HIS is mostly a parasitic surface and sits under the antenna only to reflect electromagnetic fields. Consequently, the induced current distribution on the surface strictly follows the near field of the antenna. In such cases, the HIS is commonly analysed using either transmission line model or an equivalent circuit model. Using the conventional circuit model of HIS design, it is very difficult to analyse these kind of situations because of the complex nature of the near field. Most of the works till date, reported in literature, have used an air gap (realized by foam layer) between the antenna and the HIS to isolate them [1-4]. Few works published do not use foam or spacers but conducted parametric studies or used empirical circuit models; based on parameter extraction method to design low profile antennas [5-7]. Recently, in [8], it is shown that there is a significant scope of research to address the issues related to the near field excitation of finite meta-surfaces using the Theory of Characteristic Modes (TCM). TCM is already applied to few metasurface based antenna design applications such as 
in [9] and also in metamaterial inspired structures [10, 11]. However, as per the authors' knowledge no work based on characteristic modal (CM) analysis of HIS has been reported till date. Literature review shows that HIS structures are only characterized by the transmission line modelling technique or plane wave excitation technique followed by an empirical circuit modelling [5-7]. Even though the behaviours of the most of these surfaces are described satisfactorily by circuit models, there are various situations where such predictions are very difficult [12], especially for the broadband HIS or if few number of unit cells are used to design the HIS. Moreover, in many cases, the HISs are characterized by plane wave excitation technique while in practical scenario they are excited by the near field of an antenna. As a result, the final antenna design requires rigorous simulation based on parametric studies [6]. No systematic technique is available till date to handle the near field interactions of HIS.

Present work takes up these issues and is going to use substructure based CM [13] to analyse the electromagnetic behaviour of the antenna and the HIS at close proximity. The appropriate mode of operation of the structures are identified and are synchronized at the operating frequency by controlling the antenna dimensions. Hence, the whole antenna structure is made to operate at $2.45 \mathrm{GHz}$ satisfactorily. Another advantage of using this technique is that circuit modelling of the HIS is not required as it is always a cumbersome procedure. Most importantly, circuit modelling for this present work will be very difficult because of the near field interaction between the antenna and the HIS. Unlike the conventional approach, a good physical insight of the operating modes (and also resonances) of the antenna as well as the HIS can be understood by this TCM based technique. This insight in turn is utilized in designing an HIS backed antenna with no air gap, thereby drastically reducing the antenna profile. In this process, the overall antenna footprint is also reduced with respect to the size of a conventional half wave slot at the same frequency.

\section{TCM for Multilayer Antennas}

As already discussed, the aim of the present work is to analyse a multilayer HIS backed cross slotted antenna using substructure based CM. Keeping this in mind, this section will concentrate to develop a CM analysis procedure of a conventional multilayer (three-layer) antenna structure as shown in Fig. 1 containing sandwiched layers of two dielectric slabs in between three PEC sheets which is very similar to the desired structure in this work. Subsequently, the same theory will be used to analyse the original structure. The antenna structure is analysed using the surface integral equation based Method of Moment (MoM) technique. The generalized MoM expression of the antenna structure is given in (1) where [Z] is the generalized MoM matrix, $[\mathbf{I}]$ is the coefficients of unknown MoM currents and [V] is the right hand excitation vector. The concerned MoM analysis and the extraction of
[Z] matrix are carried out using FEKO and the subsequent post-processing utilizing the $[\mathbf{Z}]$ matrix is described next. For convenience, at first, the theoretical construction of $[\mathbf{Z}]$ matrix is given and then the post-processing technique is illustrated. In (1), the MoM matrix $[\mathbf{Z}]$ is formulated by PMCHW technique [14] and the matrix [I] has every current associated with the antenna structure as shown in Fig. 1.

$$
[\mathbf{Z}]\left[\begin{array}{c}
I_{\mathrm{d}} \\
I_{\mathrm{p}} \\
I_{\mathrm{cdp}} \\
I_{\mathrm{cdm}} \\
I_{\mathrm{cdg}} \\
M_{\mathrm{d}} \\
I_{\mathrm{cp}} \\
I_{\mathrm{cg}}
\end{array}\right]=\left[\begin{array}{c}
v_{\mathrm{a}} \\
v_{\mathrm{pp}} \\
v_{\mathrm{b}} \\
v_{\mathrm{mm}} \\
v_{\mathrm{c}} \\
i \\
v_{\mathrm{e}} \\
v_{\mathrm{f}}
\end{array}\right] .
$$

The MoM current coefficients associated with the two layers of PEC marked 1 and 2 in Fig. 1 are calculated from (1) replacing all other variables in the solution vector in terms of $I_{\mathrm{p}}$ and $I_{\mathrm{cdm}}$ in (1) (as this present work is interested in finding currents on slotted antenna and on the HIS). The coefficient matrices associated with the two variables, $I_{\mathrm{p}}$ and $I_{\mathrm{cdm}}$, can be found out and equation (2) and (3) can be constructed using the same procedure as discussed in [13].

$$
\begin{gathered}
{\left[\mathbf{Z}_{\mathrm{p}}\right]\left[I_{\mathrm{p}}\right]=\left[\mathbf{V}_{\mathrm{p}}\right],} \\
{\left[\mathbf{Z}_{\mathrm{m}}\right]\left[I_{\mathrm{cdm}}\right]=\left[\mathbf{V}_{\mathrm{m}}\right] .}
\end{gathered}
$$

The coefficient matrices, $\left[\mathbf{Z}_{\mathrm{p}}\right]$ and $\left[\mathbf{Z}_{\mathrm{m}}\right]$ are used to construct the generalized eigenvalue problems (GEPs) associated with the two structures (marked 1 and 2 in Fig. 1). These matrix operators are decomposed into their Hermitian parts as shown in equations (4) and (5). For the patch (marked 1 in Fig. 1):

$$
\left[\mathbf{Z}_{\mathrm{p}}\right]=\left[\mathbf{R}_{\mathrm{p}}\right]+\mathrm{j}\left[\mathbf{X}_{\mathrm{p}}\right] \text {. }
$$

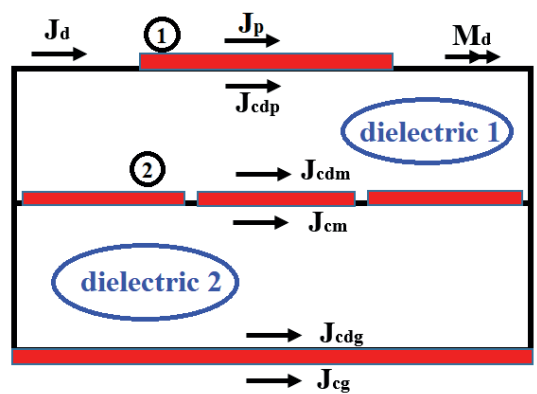

Fig. 1. Schematic diagram of a three layered HIS backed slotted antenna with MoM surface equivalent currents on the slotted antenna (Structure 1), on HIS (structure 2) and on the ground plane: $\mathbf{J}_{\mathbf{d}}=$ electric current and $\mathbf{M}_{\mathbf{d}}=$ magnetic equivalent current on the dielectric $1, \mathbf{J}_{\mathbf{p}}=$ electric current on structure 1, $\mathbf{J}_{\mathbf{c g}}=$ electric current on ground plane, $\mathbf{J}_{\mathbf{c d p}}=$ electric current between structure 1 and dielectric 1, $\mathbf{J}_{\mathbf{c d m}}=$ electric current between structure 2 and dielectric $1, \mathbf{J}_{\mathbf{c m}}=$ electric current between structure 2 and dielectric $2, \mathbf{J}_{\mathbf{c d g}}=$ electric current between ground plane and dielectric 2 
For the meta-surface (marked 2 in Fig. 1):

$$
\left[\mathbf{Z}_{\mathrm{m}}\right]=\left[\mathbf{R}_{\mathrm{m}}\right]+\mathrm{j}\left[\mathbf{X}_{\mathrm{m}}\right]
$$

The GEPs for the two structures are shown in (6) and (7):

$$
\begin{aligned}
{\left[\mathbf{X}_{\mathrm{p}}\right]\left[I_{\mathrm{n}}^{\mathbf{p}}\right] } & =\lambda_{\mathrm{n}}^{\mathrm{p}}\left[\mathbf{R}_{\mathrm{p}}\right]\left[I_{\mathrm{n}}^{\mathrm{p}}\right], \\
{\left[\mathbf{X}_{\mathrm{m}}\right]\left[I_{\mathrm{n}}^{\mathrm{m}}\right] } & =\lambda_{\mathrm{n}}^{\mathrm{m}}\left[\mathbf{R}_{\mathrm{m}}\right]\left[I_{\mathrm{n}}^{\mathrm{m}}\right],
\end{aligned}
$$

where $\lambda_{\mathrm{n}}^{\mathrm{p}}$ and $\lambda_{\mathrm{n}}^{\mathrm{m}}$ are the $n^{\text {th }}$ eigenvalue of equations (6) and (7) respectively. Similarly $I_{\mathrm{n}}^{\mathrm{p}}$ and $I_{\mathrm{n}}^{\mathrm{m}}$ are current coefficient of the $n^{\text {th }}$ mode of the patch and the HIS respectively. The surface CM current on the two structures are found out using equations (8) and (9):

$$
\begin{aligned}
& \mathbf{J}_{\mathrm{n}}^{\mathrm{p}}=\sum_{q} \mathbf{f}_{\mathrm{q}}^{\mathrm{p}} I_{\mathrm{n}, \mathrm{q}}^{\mathrm{p}}, \\
& \mathbf{J}_{\mathrm{n}}^{\mathrm{m}}=\sum_{m} \mathbf{f}_{\mathrm{q}}^{\mathrm{m}} I_{\mathrm{n}, \mathrm{q}}^{\mathrm{m}},
\end{aligned}
$$

where $\mathbf{f}_{\mathrm{q}}^{\mathrm{p}}$ and $\mathbf{f}_{\mathrm{q}}^{\mathrm{m}}$ are the $\mathrm{q}^{\text {th }}$ Rao-Wilton-Glisson [15] basis functions on the slotted antenna (marked 1 in Fig. 1) and the metasurface (marked 2 in Fig. 1) respectively. $I_{\mathrm{n}, \mathrm{q}}^{\mathrm{p}}$ and $I_{\mathrm{n}, \mathrm{q}}^{\mathrm{m}}$ are the $q^{\text {th }}$ elements of the current coefficient matrix $I_{\mathrm{n}}^{\mathrm{p}}$ and $I_{\mathrm{n}}^{\mathrm{m}}$ respectively.

\section{Characterization of HIS Using CM}

Firstly, a $3 \times 3$ HIS is characterized by using CM analysis. The meta-surface is designed on a dielectric substrate $\left(\varepsilon_{r}=10.2\right)$ having thickness $3.2 \mathrm{~mm}$ and the other dimensions are given in Fig. 2(a). The HIS structure is simulated in FEKO and the MoM [Z] matrix is extracted to compute CM currents and eigenvalues of it (for Mode 1 by an in-house code). The characteristic angle (CA) plot in Fig. 2(c) shows a $180^{\circ}$ crossing at $2.45 \mathrm{GHz}$, which corresponds to the resonance of this meta-surface structure. An X-directed plane wave excitation ( $0^{\circ}$ phase at the surface of HIS) of $\mathbf{E}_{\mathbf{i}}=1 \mathrm{~V} / \mathrm{m}$ is impinged on the surface and the phase of the reflected field $\mathbf{E}_{\mathbf{r}}$ is recorded theoretically as governed by the (10):

$$
\mathbf{E}_{\mathbf{r}}=\frac{\left\langle\mathbf{E}_{\mathbf{i}}, \mathbf{J}_{\mathbf{1}}\right\rangle}{1+\mathrm{j} \lambda_{1}} \mathbf{E}_{\mathbf{1}},
$$

where $\mathbf{E}_{\mathbf{i}}$ is the incident field, $\lambda_{1}$ is the CM eigenvalue and $\mathbf{E}_{\mathbf{1}}$ is the scattered field by the CM current $\mathbf{J}_{\mathbf{1}}$. The characteristic angle $\alpha=180^{\circ}-\tan ^{-1}\left(\lambda_{1}\right)$. It is observed that the phase angle of $\mathbf{E}_{\mathbf{r}}$ is dependent on the phase of the denominator as well as on the scattered near field $\mathbf{E}_{\mathbf{1}}$ in equation (10).

In Fig. 3, a $0^{\circ}$ phase of the reflected field is observed at $2.445 \mathrm{GHz}$ which is very close to $2.45 \mathrm{GHz}$. So at this frequency, the surface offers high impedance. Although the main aim of this present work is to use $3 \times 3$ HIS for miniaturization, CM analysis of a $5 \times 5$ HIS is conducted for the justification of the technique undertaken and the results are given in Fig. 2(b) and (c). It is noted that the $5 \times 5$ HIS with a slightly reduced sized single unit cell (than $3 \times 3$ HIS) gives the same resonant frequency. So, it can be concluded that a truncated version of HIS shows a slight increase in resonant frequency than the significantly bigger structure keeping unit cell dimension remains same. Here, the CM analysis helped to predict the resonant frequency accurately. Figure 3 shows the angle of reflected field $\mathbf{E}_{\mathbf{r}}$ over frequency in response to an incident field $\mathbf{E}_{\mathbf{i}}$ (same as discussed above) which is governed by equation (10). Although the size of the individual elements of the surface is much smaller than the wavelength (at $2.45 \mathrm{GHz}$ ) at the resonant frequency, the overall structure shows a resonance at the said frequency. As the reflected field is inversely proportional to the eigenvalue $\left(\lambda_{1}\right)$ in (10), the field becomes maximum at resonant frequency (as $\lambda$ becomes zero corresponding to the $180^{\circ}$ crossing of the characteristic angle in Fig. 2(c)). Hence, it is a parallel resonant structure and this parallel resonance property of HIS has been characterized in [16]. Here, the HIS can be characterized alternatively by CM analysis and the CM resonance frequency is the point where the surface shows high impedance. Also the CM eigenvalue is directly proportional to the difference in stored energies (electric and magnetic) and net storage changes from one kind (electric or magnetic) to the other as eigenvalue crosses zero $[17,18]$. Hence, the subsequent discussion will take the $\mathrm{CM}$ resonant frequency as the determining point for high impedance behaviour of the surface.
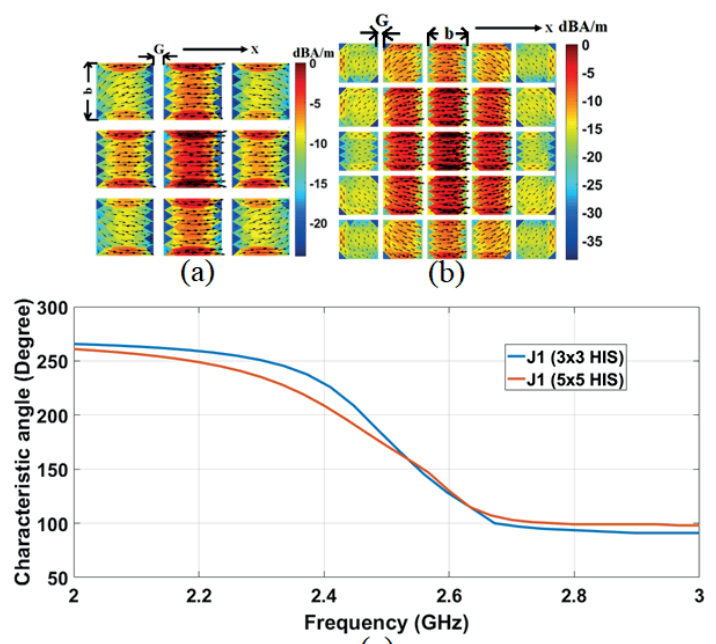

(c)

Fig. 2. (a) $\mathrm{CM}$ current mode 1 of $\mathrm{HIS}$ at $2.45 \mathrm{GHz}$ with $\mathrm{G}=2$ $\mathrm{mm}$ and $\mathrm{b}=16 \mathrm{~mm}$ of the $3 \times 3 \mathrm{HIS}$, (b) CM current at $2.45 \mathrm{GHz}$ with $\mathrm{G}=2 \mathrm{~mm}$ and $\mathrm{b}=15 \mathrm{~mm}$ of the $5 \times 5 \mathrm{HIS}$ of mode 1 , (c) Characteristic angle plot of mode 1 of these structures.

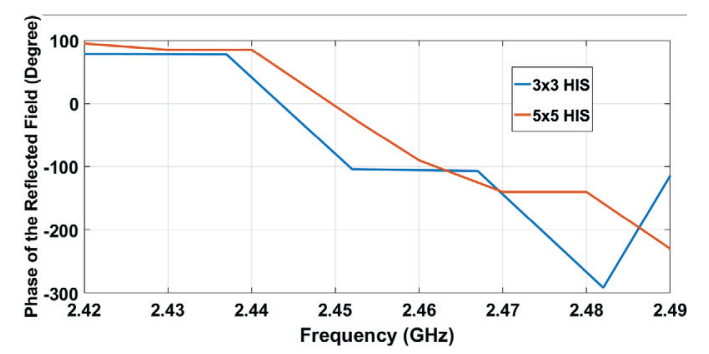

Fig. 3. Phase of reflected field from the HIS (incident field is at $0^{\circ}$ to the surface) : $3 \times 3$ HIS and $5 \times 5$ HIS 


\section{CMs of HIS Backed Antenna}

Previous section characterizes HIS by a plane wave excitation using TCM. This section is going to take up the practical problem of placing an HIS in the near field of a cross slotted antenna and uses feed independent substructure based TCM (follow the method discussed in Sec. 2) to analyse the natural modes of the whole structure. Subsequently, a proper feeding arrangement is used to excite the proper modes on the structure for achieving the design goal. The operating frequency is chosen as $2.45 \mathrm{GHz}$. It is observed that the resonant frequencies of both the cross slot and the HIS shift from that of the unloaded case (when these two structures are separately studied using CM). This happens because of the large interaction of near field between the antenna and the HIS.

\subsection{CM of a Cross Slotted Antenna Without HIS}

To begin with, the CMs of an unloaded cross slotted ground plane antenna designed on a $1.6 \mathrm{~mm}$ thick substrate $\left(\varepsilon_{\mathrm{r}}=2.4\right)$ is obtained from FEKO. The CMs of the slot antenna is obtained by taking only the electric characteristic currents on the ground plane (in Fig. 4(a) and (b)) by similar method discussed in [19]. The characteristic angle of the first two CMs of the antenna is shown in Fig. 4(c) crosses $180^{\circ}$ at $2.45 \mathrm{GHz}$ (degenerate modes). The advantage of using electric CM currents on the ground plane, is to take care of the slot and the shape of the ground plane as well. The first two CM currents are only considered because their pattern are spatially orthogonal to each other (as the aim of this work is to obtain circularly polarized $(\mathrm{CP})$ radiation from the antenna, we are interested only in the spatially orthogonal modes). In Fig. 4(a), the dimensions of the cross slotted antenna is given as $L_{\mathrm{g}}=27 \mathrm{~mm}$ and $L_{\mathrm{S}}=20 \mathrm{~mm}$.

\subsection{Loading with HIS}

This subsection is going to analyse the cross slotted antenna loaded HIS structure. The slotted antenna is placed on top of the HIS for reduction of backward radiation (thickness and permittivity of the dielectric substrate of the slotted antenna and the HIS are kept the same as discussed in Sec. 4.1 and Sec, 3). It is to be noted that a strong near field interaction takes place between the slotted antenna and the HIS as they come very close to each other. This interaction changes the resonant frequencies of the desired operating modes of both structures from that of the unloaded condition. Also, as the slotted antenna is considered as the only driving element (only it is fed), so the current distribution on the HIS is supported by the near field distribution of the slotted antenna. To address all of these issues, substructure based CM is applied to this stacked structure of antenna and HIS. Initial dimensions of the antenna and the HIS are taken as described in Sec. 4.1 and Sec. 3. The MoM [Z] matrix of the composite structure is extracted using FEKO and the substructure CMs of the slotted antenna and the HIS are found out by using the method as discussed in Sec. 2. In this case, the CMs of both structures are obtained separately even though they are strongly electromagnetically coupled to each other. It is found that the resonant frequencies of mode 1 and 2 of the antenna and mode 2 and 3 of the HIS are left shifted in frequency unevenly. To bring resonant frequencies of mode 1 and 2 of the slotted antenna to $2.45 \mathrm{GHz}$, the length of both the cross slots and the size of the square ground plane are reduced. Similarly, the size of unit cells of the HIS is reduced and the gap $(\mathrm{G})$ between these patches is varied to bring the resonant frequencies of mode 2 and 3 very close to $2.45 \mathrm{GHz}$, as the high impedance condition is required for both modes at the mentioned particular frequency.

It is evident that the CM analysis helps in understanding the modal current distributions clearly. Hence, the resonant frequencies of both structures can be properly synchronized by controlling the dimensions. For the present work, it is sufficient to take into account the first two modes of the slotted antenna as the $\mathrm{CP}$ operation requires two orthogonally spaced modes only. On the other hand, modes 2 and 3 of the HIS are the most suitable ones to be excited because current distributions of these modes have similarities with the antenna modes. The resonant frequencies of the structures are taken where the characteristic angle plot over frequency crosses at $180^{\circ}$ (or in other words Eigenvalue plot over frequency crosses at $0^{\circ}$ ). At this point, resonant frequencies of modes 1 and 2 of the slotted antenna and modes 2 and 3 of HIS are set to $2.45 \mathrm{GHz}$. Now, to achieve CP operation of the slotted antenna, the orthogonally spaced modes (1 and 2 ) need to be $90^{\circ}$ out of phase with each other. Thus, the length of the horizontal slot is increased a little bit to reduce the resonant frequency of mode 1 and the length of the vertical slot is reduced slightly to increase resonant frequency of the mode 2 as shown in Fig. 6. However, in doing so, it is observed that the mode 2 and 3 of the HIS are also going to be separated out in frequency (in Fig. 8 and 9) which is unwanted.

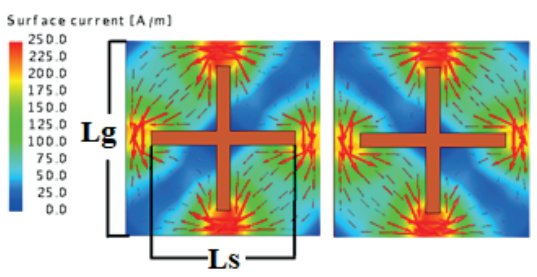

(a)

(b)

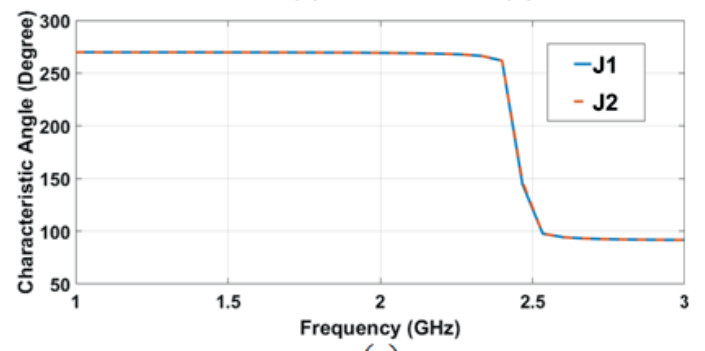

(c)

Fig. 4. Modes of the unloaded slotted antenna at $2.45 \mathrm{GHz}$ (a) Mode 1, (b) Mode 2, (c) Characteristic angle plot of mode 1 . 
Therefore, the width of the tip of the slots are increased a little bit to counter this change. Still, the resonant frequencies of modes 2 and 3 of HIS are slightly separated as evident from Fig. 8 and 9. The CM current distributions of the cross slotted antenna and the HIS are shown in Fig. 5 and Fig. 7 respectively. The eigenvalue plots are given in Fig. 8(a) and 9(a) because they are directly related to the stored energies as discussed in Section 3 and it helps to understand physically as the magnetic stored energy rises the eigenvalues rise up to a point where it is maximum and at the point where eigenvalue plot crosses $0^{\circ}$, a high impedance from the loaded HIS is expected. The CA plots are given in Fig. 8(b) and 9(b) and the first crossing point of $180^{\circ}$ is considered to be the resonant frequency in this work. As this is a feed independent method for analysis, the antenna structure is properly fed to excite the modes as discussed here and simulated in the Sec. 5 keeping all dimensions same as obtained through this discussion. The dimensions are given in Fig. 10 in Sec. 5.

\subsection{Size Reduction of the Antenna Structure}

As the CM analysis of the slotted antenna involves the currents on the ground plane (excluding the slot), it can help optimizing the overall ground plane dimension (comparable to the slots). Moreover, the substructure CM analysis for the structures (slot antenna and HIS) simplifies the design procedure and a reduced size compact antenna is obtained by properly exciting modes on the two structures with a fine tuning of the dimensions according to the resonant frequencies. The overall antenna size becomes $0.23 \lambda_{0} \times 0.23 \lambda_{0} \times 0.039 \lambda_{0}$ and the size of only the slotted patch becomes $0.16 \lambda_{0} \times 0.16 \lambda_{0}$ at $2.45 \mathrm{GHz}$ (where $\lambda_{0}$ is the free space wavelength). The area of the overall antenna size is $78 \%$ smaller and that of the patch $88 \%$ smaller than a half-wavelength antenna operating at the same frequency.

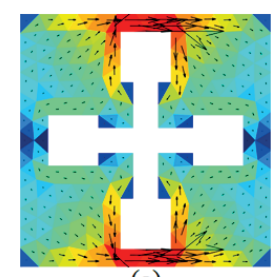

(a)

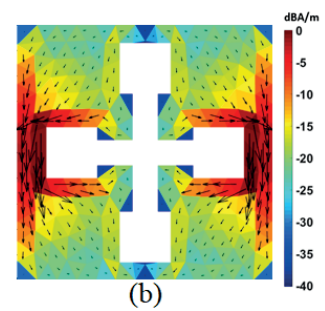

(b)
Fig. 5. Substructure CM currents on the slotted antenna at $2.45 \mathrm{GHz}$ (a) Mode 1, (b) Mode 2

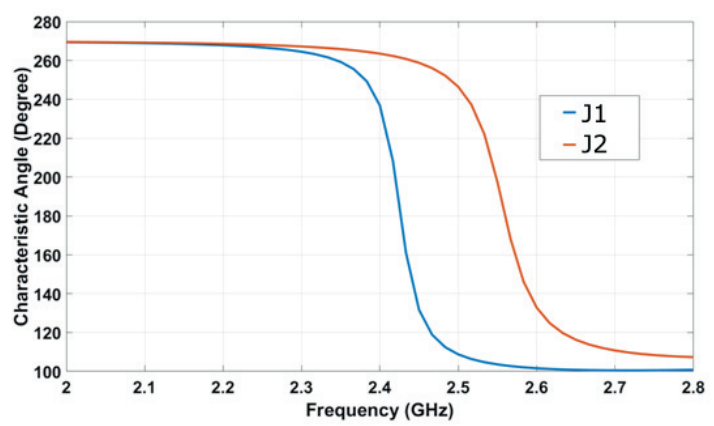

Fig. 6. Characteristic angles over frequency of the two modes of the slotted ground antenna of interest.

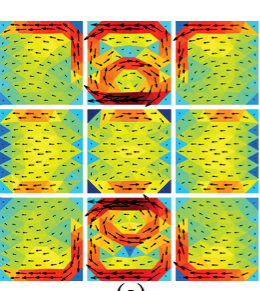

(a)

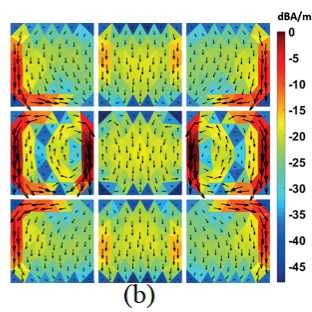

Fig. 7. Substructure CM currents on the HIS at $2.45 \mathrm{GHz}$, (a) Mode 2, (b) Mode 3

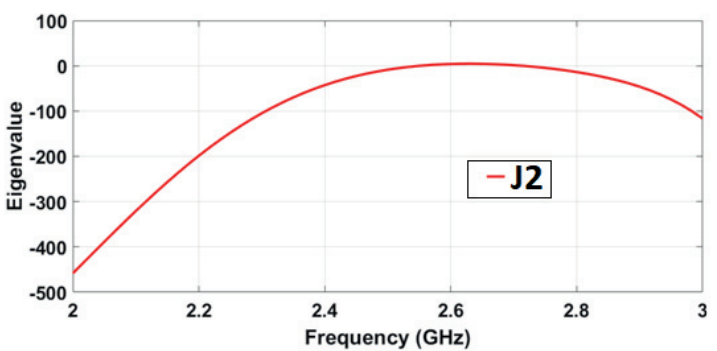

(a)

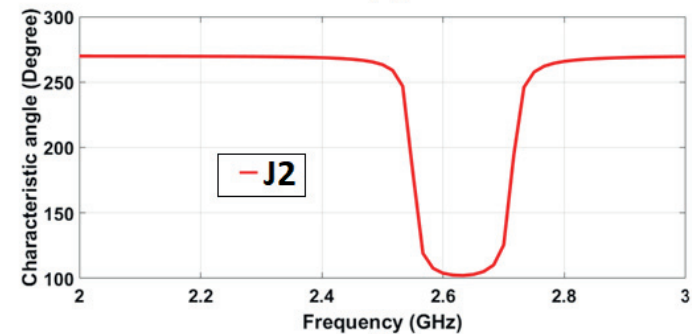

(b)
Fig. 8. Mode 2 of loaded HIS over frequency: (a) Eigenvalue, (b) Characteristic angle.

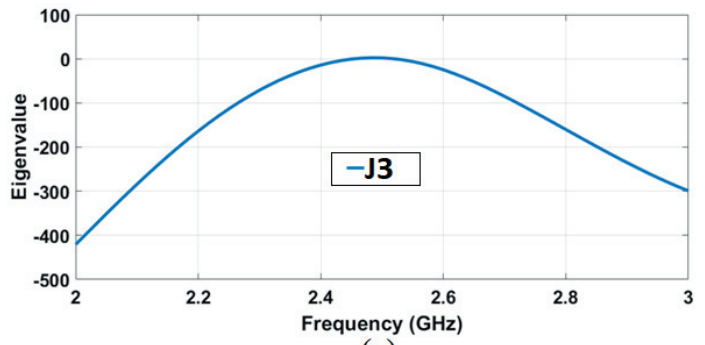

(a)

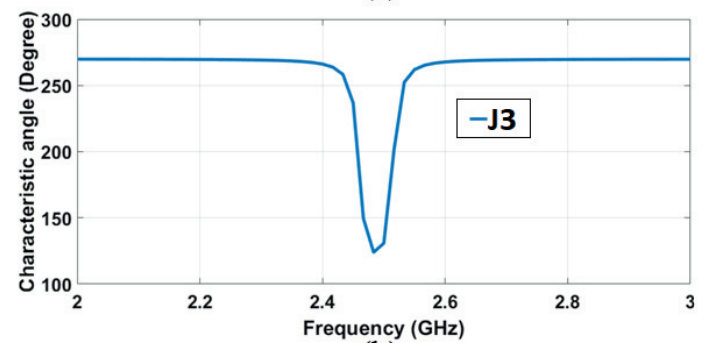

(b)

Fig. 9. Mode 3 of loaded HIS over frequency: (a) Eigenvalue, (b) Characteristic angle. 

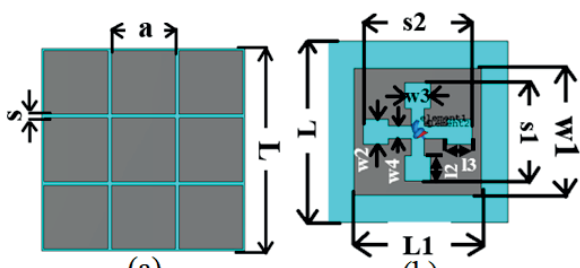

(a)

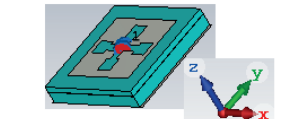

(c)

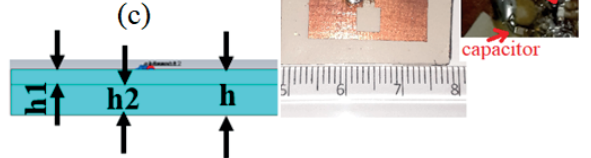

(d)

(e)

Fig. 10. Simulated antenna prototype: (a) Top view of HIS having $\mathrm{L}=28.5 \mathrm{~mm}, \mathrm{~s}=0.5 \mathrm{~mm}, \mathrm{a}=9 \mathrm{~mm}$, (b) Top view of the slotted antenna placed on the top of HIS having $\mathrm{L}=28.5 \mathrm{~mm}, \mathrm{~L} 1=\mathrm{W} 1=20 \mathrm{~mm}, \mathrm{~s} 1=15.5 \mathrm{~mm}, \mathrm{w} 3=\mathrm{w} 2=4$ $\mathrm{mm}, \mathrm{w} 4=2 \mathrm{~mm}, 12=13=4 \mathrm{~mm}, \mathrm{~s} 2=17.2 \mathrm{~mm}$, (c) Perspective view with coordinate axes (d) Side view of the antenna with $\mathrm{h} 1=1.6 \mathrm{~mm}, \mathrm{~h} 2=3.2 \mathrm{~mm}, \mathrm{~h}=4.8 \mathrm{~mm}$ ( $\varepsilon_{\mathrm{r}}=10.2$ of the substrate for the height $\mathrm{h} 2$ and $\varepsilon_{\mathrm{r}}=2.4$ for the height h1), Fabricated prototype (e) Top view (feeding location in the inset).

\section{Antenna Design}

The previous section analyses and optimizes the antenna structure without excitation. Here, the prototype antenna is simulated by using CST Microwave Studio by giving proper feeding arrangements. The design parameters of the antenna are kept the same as the outcome in Sec. 4. To get a CP radiation, both horizontal and vertical modes (mode 1 and 2 discussed in Sec. 4) on the slotted ground plane are necessary to be excited. Hence, the location of the gap feed is chosen to be at the center of both the slots where the CM currents of the two modes are minimum as evident in Fig. 5 (a) and (b) (as this structure is parallel resonant so the feeding point should be placed at the current minima). In order to match the antenna properly to $50 \Omega$, an inductor of $6 \mathrm{nH}$ in series with the feed and a capacitor of $0.5 \mathrm{pF}$ in shunt with the input port is used (both are lumped components). However the practical values of the fabricated capacitor and the inductor are $0.5 \mathrm{pF}( \pm 0.1 \mathrm{pF})$ and $6.2 \mathrm{nH}( \pm 0.3 \mathrm{nH})$ respectively.

\section{Results and Discussions}

The simulated current distribution (at $2.45 \mathrm{GHz}$ ) on the slotted ground in Fig. 11 confirms that two spatially orthogonal modes are excited. The simulated and measured plots of axial ratio show a $3-\mathrm{dB}$ bandwidth of $40 \mathrm{MHz}$ around the design frequency $2.45 \mathrm{GHz}$ in Fig. 12. The S11 (reflection coefficient) plot also shows an impedance bandwidth of approximately $100 \mathrm{MHz}$ which satisfactorily covers the entire CP band of interest in Fig. 13. The measurement results slightly deviates from the simulated ones because of the influence of the coaxial probe in the vicinity of the slotted ground. In Fig. 10(e), the coaxial cable is attached to the location of the current minimum of the ground plane (Fig. 11) of the slotted antenna so that the influence of the cable is the minimal on the current distribution on the ground plane and therefore the effect on the radiation pattern is not very significant. Some degradation is shown in Fig. 14 and 16.

The simulated and measured plots of radiation patterns on two principal vertical planes, i.e. $\phi=0^{\circ}$ and $\phi=90^{\circ}$ are shown in Fig. 14(a) and (b) respectively. Simulated patterns for both G_ph and G_th (G stands for Gain) at both planes are mostly directed upward (towards z-axis) having a Front-toBack Ratio (FBR) $4 \mathrm{~dB}$ with a realized gain of $3.96 \mathrm{dBiC}$ at the bore-sight direction. Measured radiation patterns closely resembles the simulated ones but a slightly more amount of measured back-lobe for $G_{-}$th in Fig. 14 is observed. The back radiation is decreased due to placement of the HIS at the back of the slotted antenna than that if the HIS is absent. The back radiation cannot be significantly reduced since the electromagnetic waves are diffracted through the edges of the finite HIS structure. Notice that in Fig. 7(a) and (b) the edge elements of the HIS has significant CM current components which are excited due to the induced near field produced by the current distribution patterns of the slotted ground plane. The 3-dB beam-width of the antenna is observed to be approximately $120^{\circ}$. In Fig. 14 and 16 , both G_th and G_ph components of radiated fields are shown to clearly understand their variations over the entire beam-width as well as over the total angular range as the axial ratio is recorded at the bore-sight direction only. Fig. 15 (a) and (b) show that the CP radiation is $\mathrm{LHCP}$ in nature at the broadside direction (within the $3-\mathrm{dB}$ ). The RHCP component at broadside direction is below $20 \mathrm{~dB}$ at $\phi=0^{\circ}$ plane and below $15 \mathrm{~dB}$ at $\phi=90^{\circ}$ plane. An absorbing paint [21] is painted at the edges and the back side of the bottom ground plane to improve the FBR slightly. Fig. 16 shows a 5 dB FBR of the antenna after applying such a absorbing paint. The overall antenna structure is $28.5 \mathrm{~mm} \times 28.5 \mathrm{~mm} \times 4.8 \mathrm{~mm}$ at $2.45 \mathrm{GHz}$. Other works related to the HIS backed CP slotted antennas, are having either the overall antenna dimensions larger than that reported in this present work or having complicated design procedure or having less amount of gain than the present work. In Tab. 1 the performance of the antenna presented in this work is compared with the relevant works found in literature.

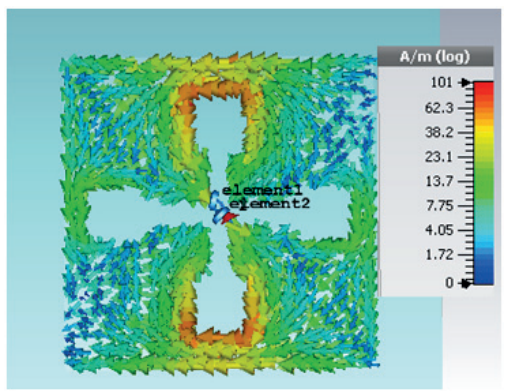

Fig. 11. Simulated current distribution on the slotted antenna structure at $2.45 \mathrm{GHz}$ in CST Microwave studio at a particular instant. 


\begin{tabular}{|c|c|c|c|c|c|}
\hline $\begin{array}{c}\text { Referenced } \\
\text { Antennas }\end{array}$ & Frequency $(\mathrm{GHz})$ & $\begin{array}{l}\text { Antenna volume }\left(\mathrm{mm}^{3}\right) \\
\left.\text { (in trems of } \lambda_{0}\right)\end{array}$ & $\begin{array}{l}\text { 10-dB impedance } \\
\text { BW }(\%)\end{array}$ & $\begin{array}{l}\text { 3-dB Axial } \\
\text { ratio BW }(\%)\end{array}$ & Gain (dBi) \\
\hline$[5]$ & 2.58 & $40 \times 40 \times 3\left(0.34 \lambda_{0} \times 0.34 \lambda_{0} \times 0.025 \lambda_{0}\right)$ & 4.6 & 1.46 & 2.98 \\
\hline$[6]$ & 2.5 & $35 \times 35 \times 7.9\left(0.29 \lambda_{0} \times 0.29 \lambda_{0} \times 0.065 \lambda_{0}\right)$ & 8 & - & 4.5 \\
\hline$[20]$ & 2.45 & $35.5 \times 35.5 \times 3.7\left(0.29 \lambda_{0} \times 0.29 \lambda_{0} \times 0.03 \lambda_{0}\right)$ & 5.2 & 1.6 & 3.41 \\
\hline Our work & 2.45 & $28.5 \times 28.5 \times 4.8\left(0.23 \lambda_{0} \times 0.23 \lambda_{0} \times 0.039 \lambda_{0}\right)$ & 4.4 & 1.46 & $3.96 \mathrm{dBiC}$ \\
\hline
\end{tabular}

Tab. 1. Comparison table for miniaturized antennas with the present work.

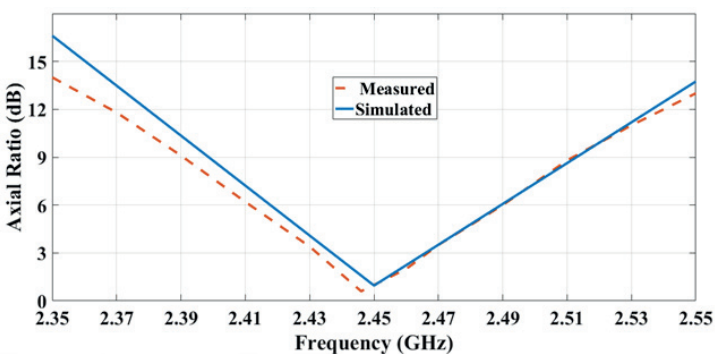

Fig. 12. Simulated and measured plots of axial ratio.

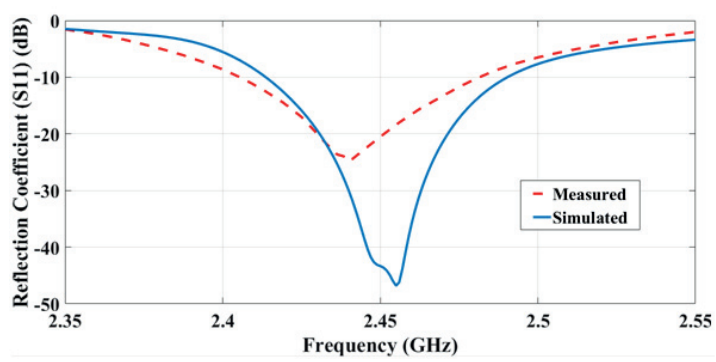

Fig. 13. Simulated and measured plots of reflection coefficient at the feed of the slotted antenna.

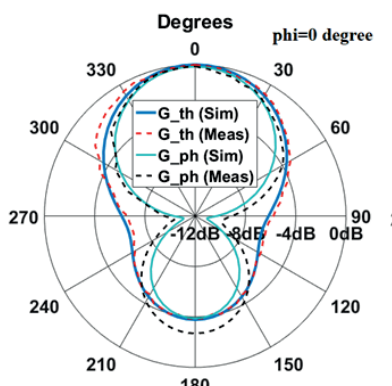

180

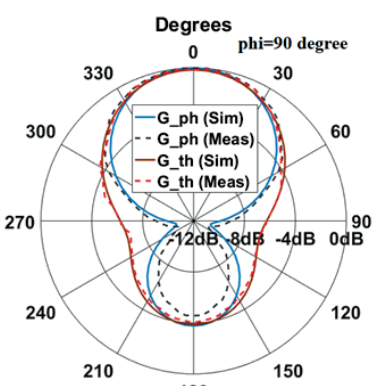

(b)
Fig. 14. Simulated and measured normalized gain plots on two orthogonal vertical planes at $2.45 \mathrm{GHz}$, (a) $\phi=0^{\circ}(\mathrm{x}-\mathrm{z}$ plane), (b) $\phi=90^{\circ}$ (y-z plane)

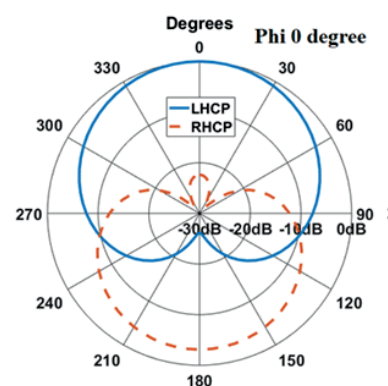

(a)

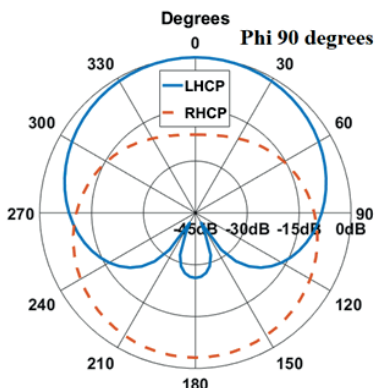

(b)
Fig. 15. Simulated normalized plots for left and right hand circular polarization components of gain pattern at two principal vertical planes at $2.45 \mathrm{GHz}$, (a) $\phi=0^{\circ}$, (b) $\phi=90^{\circ}$.

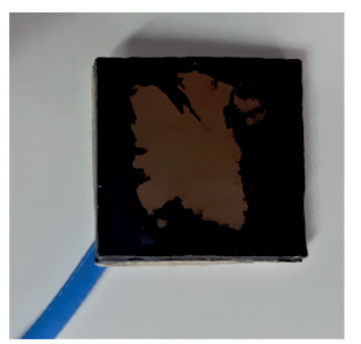

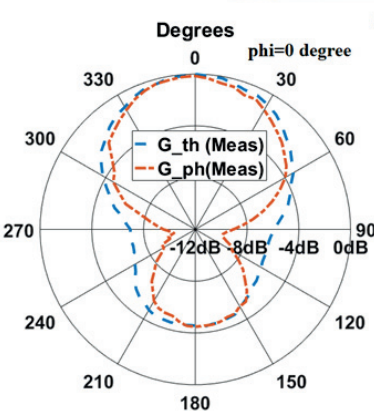

(b)

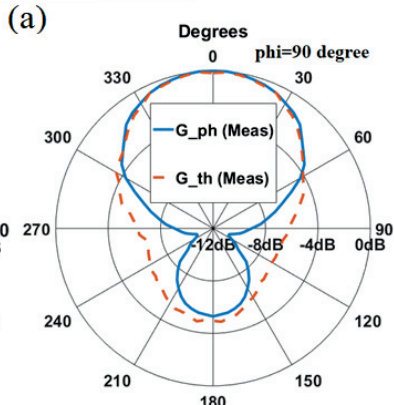

(c)
Fig. 16. (a) Ground plane edges coated with absorbing paint, Simulated and measured normalized gain plots on two orthogonal vertical planes at $2.45 \mathrm{GHz}$ with absorbing paint, (b) $\phi=0^{\circ}$ (x-z plane), (b) $\phi=90^{\circ}$ (y-z plane).

\section{Conclusion}

This work illustrates how a high impedance surfacebacked cross slotted antenna can be analysed and designed using the theory of characteristic modes without any empirical circuit modelling. The near field effect of the antenna on the high impedance surface is taken into consideration. Thereby, the overall antenna dimensions are minimized and a circularly polarized radiation is achieved by using the knowledge of the characteristic modal current distribution on the different segment of the structure. The proposed antenna is small sized, light weight and low profile suitable for application in RFID tags. Also, novel dual and multi-band polarization diversity antennas can be studied and designed more systematically using the proposed method.

\section{Acknowledgments}

Arka Bhattacharyya thankfully acknowledges Council of Scientific and Industrial Research (CSIR), India for financial support under File No. 09/096(0882)/2017-EMR-I dated 31.03.2017. 


\section{References}

[1] CAI, Y., LI, K., YIN, Y., et al. Dual-band circularly polarized antenna combining slot and microstrip modes for GPS with HIS ground plane. IEEE Antennas and Wireless Propagation Letters, 2015, vol. 14, p. 1129-1132. DOI: 10.1109/LAWP.2015.2395538

[2] FENG, D., ZHAI, H., XI, L., et al. A broadband low-profile circular-polarized antenna on an AMC reflector. 2017, IEEE Antennas and Wireless Propagation Letters, vol. 16, p. 2840-2843. DOI: $10.1109 /$ LAWP.2017.2749246

[3] ZHU, J., LI, S., LIAO, S., et al. Wideband low-profile highly isolated MIMO antenna with artificial magnetic conductor. IEEE Antennas and Wireless Propagation Letters, 2018, vol. 17, no. 3, p. 458-462. DOI: 10.1109/LAWP.2018.2795018

[4] JOUBERT, J., VARDAXOGLOU, J. C., WhitTOW, W. G., et al CPW-fed cavity-backed slot radiator loaded with an AMC reflector. IEEE Transactions on Antennas and Propagation, 2012, vol. 60, no. 2, p. 735-742. DOI: 10.1109/TAP.2011.2173152

[5] DONG, Y., TOYAO, H., ITOH, T. Compact circularly-polarized patch antenna loaded with metamaterial structures. IEEE Transactions on Antennas and Propagation, 2011, vol. 59, no. 11, p. 4329-4333. DOI: 10.1109/TAP.2011.2164223

[6] AGARWAL, K., NASIMUDDIN, ALPHONES, A. RIS-based compact circularly polarized microstrip antennas. IEEE Transactions on Antennas and Propagation, 2013, vol. 61, no. 2, p. 547-554. DOI: 10.1109/TAP.2012.2225816

[7] GUPTA, G., HARISH, A. R. Performance of a dipole placed above a novel double layered via-less high impedance surface. IET Microwaves, Antennas \& Propagation, 2017, vol. 11, no. 11, p. 1609-1615. DOI: 10.1049 /iet-map.2016.0882

[8] LIN, F. H., LI, T., CHEN, Z. N. Recent progress in metasurface antennas using characteristic mode analysis. In Proceedings of the 13th European Conference on Antennas and Propagation (EuCAP). Krakow (Poland), 2019, p. 1-5. ISBN: 978-88-907018-8-7

[9] SALIH, A. A., CHEN, Z. N., MOUTHAAN, K. Characteristic mode analysis and metasurface- based suppression of higher order modes of a $2 \times 2$ closely spaced phased array. IEEE Transactions on Antennas and Propagation, 2017, vol. 65, no. 3, p. 1141-1150. DOI: 10.1109/TAP.2016.2647683

[10] CHUKWUKA, O., SEETHARAMDOO, D., RABAH, M. H. Coupling analysis of metamaterial inspired structures using the theory of characteristic modes. In Proceedings of the 13th European Conference on Antennas and Propagation (EuCAP). Krakow (Poland), 2019, p. 1-5. ISBN: 978-88-907018-8-7

[11] RABAH, M. H., SEETHARAMDOO, D., BERBINEAU, M. Analysis of miniature metamaterial and magnetodielectric arbitraryshaped patch antennas using characteristic modes: Evaluation of the q factor. IEEE Transactions on Antennas and Propagation, vol. 64 no. 7 p. 2719-2731. DOI: 10.1109/TAP.2016.2571723

[12] FLUHLER, H. U. Ultra-wideband (UWB) artificial magnetic conductor (AMC) metamaterials for electrically thin antennas and arrays. US Patent 8451189B1, 2013.

[13] BHATTACHARYYA, A., GUPTA, B. On the size reduction of slotted finite ground plane of a circularly polarized microstrip patch antenna using substructure characteristic modes. In Proceedings of the 13th European Conference on Antennas and Propagation (EuCAP). Krakow (Poland), 2019, p. 1-5. ISBN: 978-88-907018-8-7
[14] KISHK, A., SHAFAI, L. Different formulations for numerical solution of single or multibodies of revolution with mixed boundary conditions. IEEE Transactions on Antennas and Propagation, 1986, vol. 34, no. 5, p.666-673. DOI: 10.1109/TAP.1986.1143875

[15] RAO, S., WILTON, D., GLISSON, A. Electromagnetic scattering by surfaces of arbitrary shape. IEEE Transactions on Antennas and Propagation, 1982, vol. 30, no. 3, p. 409-418. DOI: 10.1109 /TAP.1982.1142818

[16] SIEVENPIPER, D., ZHANG, L., BROAS, R. F. J., et al. Highimpedance electromagnetic surfaces with a forbidden frequency band. IEEE Transactions on Microwave Theory and Techniques, 1999, vol. 47, no. 11 , p. 2059-2074. DOI: 10.1109/22.798001

[17] HAZDRA, P., CAPEK, M., MASEK, M., et al. An introduction to the source concept for antennas. Radioengineering, 2016, vol. 25, no. 1, p. 12-17. DOI: $10.13164 /$ re.2016.0012

[18] SCHAB, K., JELINEK, L., CAPEK, M., et al. Energy stored by radiating systems. IEEE Access, 2018, vol. 6, p. 10553-10568. DOI: 10.1109/ACCESS.2018.2807922

[19] BHATTACHARYYA, A., GUPTA, B. Investigations on effects of finite ground plane on slot antennas using characteristic modes. In Proceedings of the IEEE International Symposium on Antennas and Propagation \& USNC/URSI National Radio Science Meeting. San Diego (USA), 2017, p. 169-170. DOI: 10.1109/APUSNCURSINRSM.2017.8072127

[20] VANDELlE, E., VUONG, T., ARDILA, G., et al. Miniaturized antenna on a paper substrate. In Proceedings of the 49th European Microwave Conference (EuMC). Paris (France), 2019, p. 73-76. DOI: 10.23919/EuMC.2019.8910815

[21] IWAMARU, T., KATSUMATA, H., UEKUSA, S., et al. Development of microwave absorbing materials prepared from a polymer binder including japanese lacquer and epoxy resin. Physics Procedia, 2012, vol. 23, p. 69-72. DOI: 10.1016/j.phpro.2012.01.018

\section{About the Authors ...}

Arka BHATTACHARYYA (corresponding author) was born in May 1989. He received his M.E. from Jadavpur University, Kolkata in 2014. His research interests include computational electromagnetics and characteristic mode technique in antenna design.

Kaushik PATRA He received his Ph.D. from Jadavpur University, Kolkata in 2019. He is currently an Assistant Professor in the Institute of Engineering and Management, Kolkata His research interests include approximate modelling for microstrip lines for EMC calculation and application in travelling wave and leaky wave antennas.

Bhaskar GUPTA was born in March 1960. He is currently a Professor in Jadavpur University. His research interests include planar and dielectric resonator antennas and MEMS based antenna,wearable antennas, computational electromagnetics. 